\section{Exploring the Reactivity of 2-Trichloromethylbenzoxazoles for Access to Substituted Benzoxazoles}

Roy P. Lester, ${ }^{\dagger}$ Travis Bham, ${ }^{\S}$ Thomas $\mathrm{W}_{*}$. Bousfield, ${ }^{\S}$ William Lewis, ${ }^{\dagger}$ Jason E Camp ${ }^{*, \dagger}, \S$

${ }^{\dagger}$ School of Chemistry, University of Nottingham, Nottingham, U.K.

${ }^{\S}$ Department of Chemical Sciences, University of Huddersfield, Queensgate, Huddersfield, U.K.

j.e.camp@hud.ac.uk

RECEIVED DATE (will be automatically inserted after manuscript is accepted).

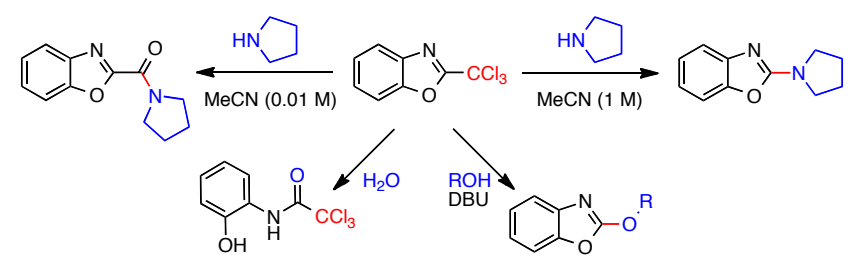

The reactivity of 2-trichloromethylbenzoxazoles towards various nucleophiles, under metal free or iron-catalyzed conditions, for the synthesis of substituted benzoxazoles is described. These methods allow for selective substitution at either the 2- or 2'- position of the benzoxazoles using the same starting materials / reagents. This approach allows for the controlled synthesis of a variety of key derivatives from a single 2-trichloromethylbenzoxazole starting material.

The benzoxazole motif has been incorporated into a number of pharmaceuticals, agrochemicals and materials that are vital to our everyday lives. ${ }^{1}$ Due to the importance of benzoxazoles, widespread research has been conducted on their synthesis ${ }^{2}$ and subsequent functionalization. ${ }^{3}$ The selective synthesis of 2-amino- or 2-amidobenzoxazoles from both activated substrates $^{4,5}$ and via $\mathrm{C}-\mathrm{H}^{6,7}$ activation has been extensively developed (Scheme $1 \mathrm{a} / \mathrm{b}){ }^{4 a, 5 b}$ Due to the nature of the methods used for their synthesis either the 2-amino- or 2amidobenzoxazoles can be accessed, but generally not both from the same starting materials using the same reagents. Additionally, in order to mitigate the negative environmental and cost implications of noble metal catalysts, there has been a recent push to develop metal free or base metal-catalyzed methods. ${ }^{8,9}$ Thus an opportunity exists to develop selective synthetic methods for the derivatization of this important scaffold. Previously, we showed that 2trichloromethylbenzoxazoles $\mathbf{1}$ could be synthesized via a mild, metal free reaction between trichloroacetonitrile ${ }^{10}$ and 2 aminophenols 4 in methanol at $40{ }^{\circ} \mathrm{C}$ ( $c f$. Scheme 2). ${ }^{1,12}$ Whilst the reactivity of some 2-trichloromethylazoles has been investigated, ${ }^{13}$ little work on the reactivity of 2- trichloromethylbenzoxazoles has been reported. ${ }^{14}$ Herein we report our findings into controlling the reactivity of 2trichloromethylbenzoxazoles for the selective synthesis of 2amino- or 2-amidobenzoxazoles as well as other derivatives in which the carbon of the trichloromethyl moiety is either extruded or retained (Scheme 1c).

\section{Metal free synthesis of 2-aminobenzoxazoles (a) ${ }^{4 a}$}<smiles>Clc1nc2c(o1)C=C[R]C=C2</smiles>
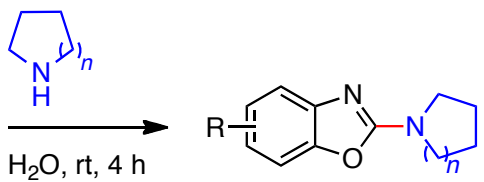

Metal free synthesis of 2-amidobenzoxazoles $(b)^{5 b}$

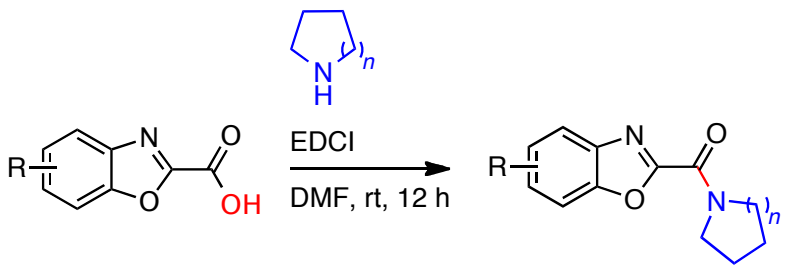

This Work - Selective synthesis of 2-amino- or 2-amidobenzoxazoles from 2-trichloromethylbenzoxazoles (c)

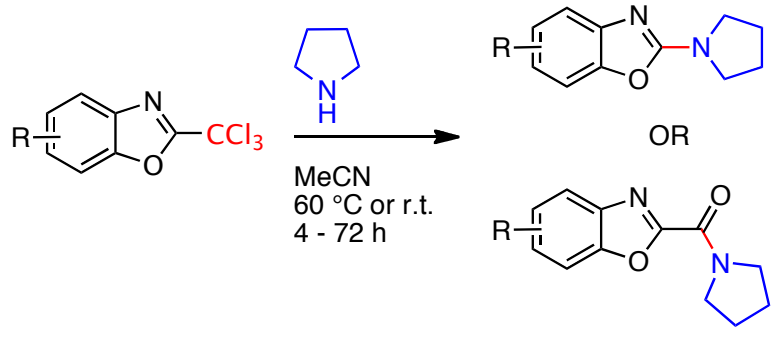

Scheme 1. Methods of benzoxazole functionalization

Due to the importance of 2-aminobenzoxazoles, the study began with the direct displacement of the 2-trichloromethyl moiety from 2-(trichloromethyl)benzo[ $d]$ oxazole $\mathbf{1}$ with an amine nucleophile. ${ }^{15}$ Initial reactions resulted in a mixture of 2-amino- and 2-amidobenzoxazoles. After screening a variety of Lewis acids, which were hoped to activate the C-2 position towards nucleophilic aromatic substitution, as well as solvents, temperatures and times it was found that the key parameters for controlling the product distribution between amine $\mathbf{2}$ and amide 5 were the molarity of the system and water content. ${ }^{16}$ Thus, reaction of 2-trichloromethylbenzoxazole $\mathbf{1}$ with pyrrolidine ( 1.1 equiv.) in dry acetonitrile $(1 \mathrm{M}$, relative to the benzoxazole) at $60{ }^{\circ} \mathrm{C}$ for $4 \mathrm{~h}$ afforded the desired 2(pyrrolidin-1-yl)benzo[ $d]$ oxazole (2) in excellent yield. The reaction could also be run at $\mathrm{rt}$, though required $13 \mathrm{~h}$ to go to completion, or in the presence of the nucleophilic catalysts DABCO ${ }^{17}$ which led to slightly improved yields ( $c f$. Scheme 3 ). Interestingly, based on $\mathrm{pKaH}$ values the reaction should 

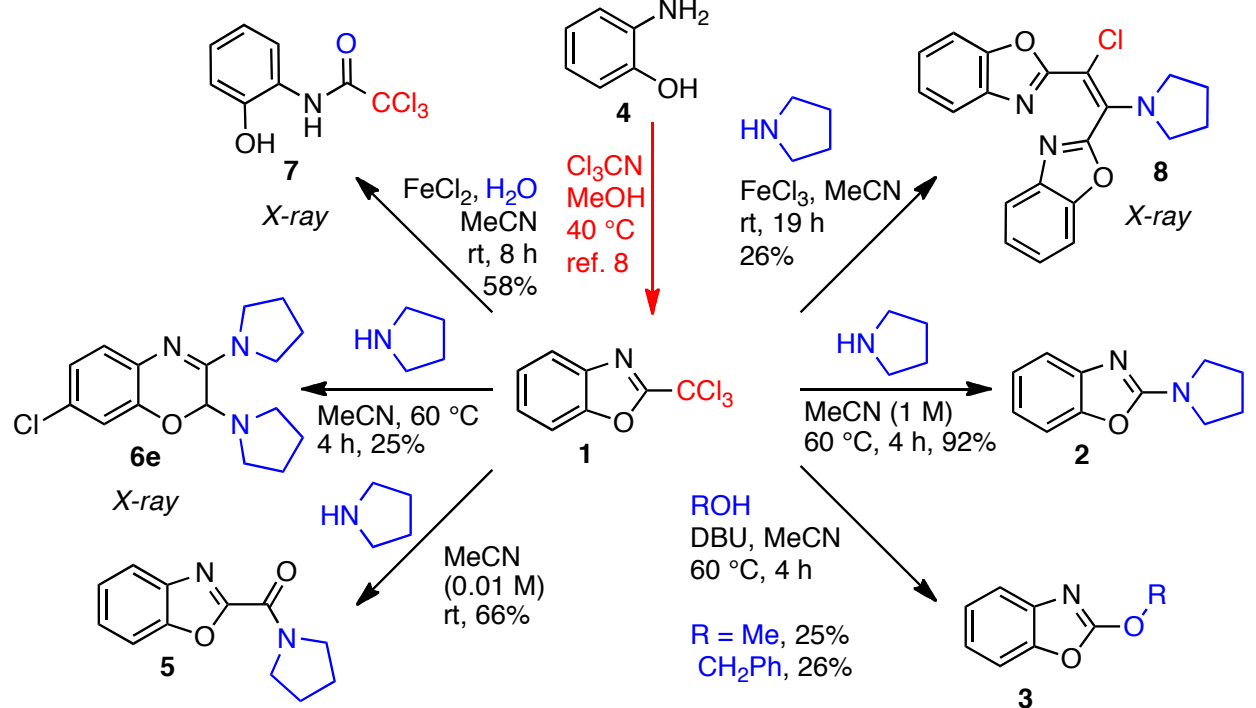

Scheme 2. DOS approach to the functionalization of 2-(trichloromethyl)benzo[d]oxazole (1)

not proceed ( $c f$. Scheme S1). ${ }^{18}$ It is believed that the liberated chloroform decomposes under the reaction conditions to form a reactive carbene intermediate, which then decomposes and thus shifts the reaction equilibrium towards the 2 -amino product 2 . $^{19}$

Building upon the selective C-2 substitution of an amine nucleophile, the addition of an alkoxide, ${ }^{20}$ generated in situ, to the 2-position of the trichloromethylbenzoxazole was investigated. It was found that methanol and benzyl alcohol could add effectively to 2-(trichloromethyl)benzo[ $d]$ oxazole (1) in the presence of DBU in acetonitrile to form either 2methoxybenzo $[d]$ oxazole (3a) or 2-(benzyloxy)benzo$[d]$ oxazole (3b), albeit in moderate yields. It was previously reported that strong bases can be used in the addition of alcohols to related trichloromethylazoles. ${ }^{13}$ Unfortunately, these conditions did not lead to an improved yield of the desired addition adducts.

Having established methods for the direct $\mathrm{C}-2$ substitution of 2-trichloromethylbenzoxazoles, the selective C-2' substitution processes were investigated. ${ }^{21}$ After screening a variety of conditions ${ }^{16}$ it was found that reaction of 2 (trichloromethyl)benzo[d]oxazole (1) with pyrrolidine $(0.9$ equiv.) in wet acetonitrile $(0.01 \mathrm{M}$, relative to the benzoxazole) at $\mathrm{rt}$ for $76 \mathrm{~h}$ afforded the desired benzo $[d]$ oxazol-2-yl(pyrrolidin-1-yl)methanone (5) in $66 \%$ yield. Reducing the reaction time to $19 \mathrm{~h}$ gave the product in $35 \%$ yield. These mild conditions are in contrast to related amide forming processes from heterocyclic trichloromethyl groups that use strong bases, high reaction temperatures or toxic solvents to achieve similar transformations. ${ }^{20}$ They therefore should be particularly applicable to substrates that are base or thermally unstable. It was also possible to reduce the reaction time via the addition of $\mathrm{FeCl}_{3}$ (1.0 equiv.), which resulted in the formation of benzo[ $[d]$ oxazol-2-yl(pyrrolidin-1yl)methanone (5) after $19 \mathrm{~h}$ in $57 \%$ yield.

As part of a substrate scope study (vide infra) it was found that the reaction of 2-(trichloromethyl)benzo[d]oxazole (1) with pyrrolidine (1.1 equiv.) in acetonitrile ( $1 \mathrm{M}$ relative to the pyrrolidine) at $60{ }^{\circ} \mathrm{C}$ for $4 \mathrm{~h}$ afforded 7-chloro-2,3di(pyrrolidin-1-yl)-2H-benzo[b][1,4] oxazine $(\mathbf{6 e})$, the structure of which was confirmed by X-ray analysis. ${ }^{22}$ Gauß and Heitzer previously reported a related ring expansion process, though they started from 2-dichloromethylbenzoxazoles rather than 2trichloromethylbenzoxazole 1. ${ }^{23}$ Also, Molinski et al. found that 2-substituted oxazolines could be converted to dihydrooxazinones in the presence of $\mathrm{SeO}_{2}{ }^{24}$ Interestingly, compound $\mathbf{6 e}$ could only be formed if a reduction was taking place under the reaction conditions, though at which point in the process the reduction is taking place is unclear. In related work, it was also shown that 2-dichloromethylbenzoxazoles can undergo hydrogen / deuterium exchange in the presence of triethylamine.

Furthermore, hydrolysis of 2-trichloromethylbenzoxazole 1 was examined (Scheme 2). It was found that upon treatment of 2-(trichloromethyl)benzo[d]oxazole (1) with $\mathrm{FeCl}_{2}(2 \mathrm{~mol} \%)$ and $\mathrm{H}_{2} \mathrm{O}$ (2.5 equiv. $)^{25}$ that ring opened 2,2,2-trichloro- $\mathrm{N}$-(2hydroxyphenyl) acetamide (7) was formed in good yield. The connectivity of acetamide 7 was confirmed by X-ray analysis. ${ }^{22}$ Acetamide 7 and related analogues have previously been shown to have germination inhibition activity against lettuce seeds. ${ }^{26}$ A screen of hydrolysis condition was undertaken to investigate this process further. It was found that the best non-metal containing process for the hydrolysis was to heat 2-trichloromethylbenzoxazole 1 in $\mathrm{H}_{2} \mathrm{O}$ at $150{ }^{\circ} \mathrm{C}$ for $2 \mathrm{~h}$. This method gave the desired 2,2,2-trichloro- $\mathrm{N}$-(2hydroxyphenyl) acetamide (7) in a moderate $37 \%$ yield. $^{22}$ 
In efforts to optimize the addition reactions and control the C-2 vs. C-2' selectivity a variety of Lewis acids were screened. ${ }^{22}$ During the course of this study it was found that the reaction of 2-trichloromethylbenzoxazole (1) with pyrrolidine (1.1 equiv.) in the presence of $\mathrm{FeCl}_{3}$ afforded bisbenzoxazole $\mathbf{8}$ in moderate yield. The structure of this novel compound was confirmed by $2 \mathrm{D}$ NMR analysis and Xray crystallography. ${ }^{22}$

With the optimized conditions in hand for the synthesis of 2-aminobenzoxazoles, the effect of substitution on the benzoxazole ring was investigated to assess the effect of electronic factors on the formation of 2-aminobenzoxazoles 2 from 2-trichloromethylbenzoxazoles 1 (Scheme 3). Thus, reaction of 2-(trichloromethyl)benzo[ $[d]$ oxazole (1a) with pyrrolidine (1.1 equiv.) at either $60{ }^{\circ} \mathrm{C} / \mathrm{rt}$ afford 2(pyrrolidin-1-yl)benzo[d]oxazole (2a) in excellent yield. The addition of the nucleophilic catalyst DABCO (10 mol \%) lead to a slight increase in yield of 2-aminobenzoxazole $2 a^{17}$ The annulation of an aromatic ring to the starting material lead to an increased yield of 2-(pyrrolidin-1-yl)naphtho[2,3-d] oxazole (2b). Interestingly, having an electron rich methoxy moiety in the system resulted in a decrease in yield of $\mathbf{2 c}$ relative to the electron deficient 5- and 6-chloro derivatives, 2d and 2e. As mentioned previously, the mass balance in the reaction of the chloro-substituted benzoxazoles 1d / 1e are the ring opened species 6d / 6e (cf. Scheme 2).

In conclusion, an investigation into the reactivity of 2trichloromethylbenzoxazole towards various nucleophiles under metal free or iron-catalyzed conditions was conducted. The newly developed methods allow for the controlled synthesis of a variety of benzoxazole derivatives starting from the same starting material. Importantly, these methods allow for substitution at either the 2- or 2'- position of the benzoxazoles by simply altering the reactant concentration / water content. Thus a variety of derivatives can be synthesized from a simple 2-trichloromethylbenzoxazole starting material via this diversity-oriented synthesis (DOS) ${ }^{27}$ type approach.

\section{EXPERIMENTAL SECTION}

General Remarks. Unless otherwise indicated, all commercially available reagents and solvents were used directly from the supplier without further purification. Solvents used for column chromatography were of technical grade. Column chromatography was performed on silica gel (60-120) mesh. Visualization was accomplished with UV light and a potassium permanganate solution. ${ }^{1} \mathrm{H}$ NMR and ${ }^{13} \mathrm{C}$ NMR were recorded at ambient temperature using $\mathrm{CDCl}_{3}$ (7.26 ppm). Chemical shift values are expressed as parts per million (ppm) and $J$ values are in Hertz. Splitting patterns are indicated as s: singlet, $\mathrm{d}$ : doublet, t: triplet, q: quartet or combination, br.s broad singlet or m: multiplet. The melting points reported are uncorrected. Infrared spectra were recorded as diluted solutions, in spectroscopic grade chloroform unless otherwise stated. Absorption maxima $(\lambda$ max) of major peaks are reported in wavenumbers (cm-1), quoted to the nearest integral wavenumber. Reactions were run in a sealed microwave reaction vessel and heated using an aluminium block on a hot plate.
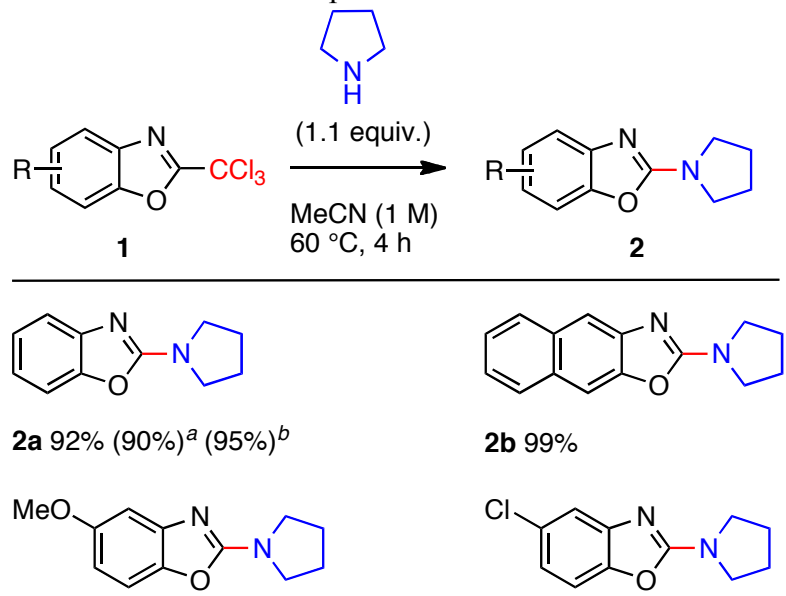

2b $99 \%$

2c $69 \%$

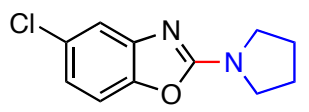

2d $81 \%$

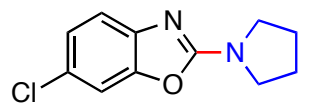

2 e $75 \%$

${ }^{a} \mathrm{rt}, 13 \mathrm{~h}^{b} \mathrm{DABCO}(10 \mathrm{~mol} \%), 60^{\circ} \mathrm{C}, 4 \mathrm{~h}$

Scheme 3. Addition of pyrrolidine to substituted 2trichloromethylbenzoxazoles

2-(Pyrrolidin-1-yl)benzo $[\boldsymbol{d}]$ oxazole $(\mathbf{2 a})^{28}$ Method $A$ : To a stirred solution of 2-(trichloromethyl)benzo $[d]$ oxazole (1a, $200 \mathrm{mg}, 0.8 \mathrm{mmol})$ in dry acetonitrile $(1 \mathrm{M})$ at r.t. was added pyrrolidine $(79 \mu \mathrm{L}, 0.9 \mathrm{mmol})$. The resultant solution was stirred at $60{ }^{\circ} \mathrm{C}$ for $4 \mathrm{~h}$. The solvent was removed in vacuo and the residue was purified by flash column chromatography on silica gel (5\% ethyl acetate in petroleum ether) to afford 2(pyrrolidin-1-yl)benzo[ $d$ ] oxazole (2a, $146 \mathrm{mg}, 92 \%$ yield) as a yellow solid. Method B: To a stirred solution of 2(trichloromethyl)benzo[d]oxazole (1a, $200 \mathrm{mg}, 0.8 \mathrm{mmol})$ in dry acetonitrile $(1 \mathrm{M})$ at r.t. was added pyrrolidine $(79 \mu \mathrm{L}, 0.9$ mmol). The resultant solution was stirred at r.t. for $13 \mathrm{~h}$. The solvent was removed in vacuo and the residue was purified by flash column chromatography on silica gel (5\% ethyl acetate in petroleum ether) to afford 2-(pyrrolidin-1yl)benzo[ $d$ ] oxazole (2a, $143 \mathrm{mg}, 90 \%$ yield) as a yellow solid. Method $C$ : To a stirred solution of 2(trichloromethyl)benzo[d] oxazole (1a, $100 \mathrm{mg}, 0.42 \mathrm{mmol})$ in dry acetonitrile $(1 \mathrm{M})$ at r.t. were added pyrrolidine $(40 \mu \mathrm{L}$, $0.47 \mathrm{mmol}$ ) and DABCO $(4.7 \mathrm{mg}, 0.042 \mathrm{mmol})$. The resultant solution was stirred at $60{ }^{\circ} \mathrm{C}$ for $4 \mathrm{~h}$. The solvent was removed in vacuo and the residue was purified by flash column chromatography on silica gel $(5 \%$ ethyl acetate in petroleum ether) to afford 2-(pyrrolidin-1-yl)benzo $[d]$ oxazole (2a, 75 mg, $95 \%$ yield) as a yellow solid. m.p. $133-135{ }^{\circ} \mathrm{C}$ (lit. m.p. $\left.136-137{ }^{\circ} \mathrm{C}\right) ; R_{\mathrm{f}}=0.25$ (1:9 ethyl acetate:petroleum ether); IR $\left(\mathrm{CHCl}_{3}\right) \mathrm{n}\left(\mathrm{cm}^{-1}\right) 3011,2978,2880,1648 ;{ }^{1} \mathrm{H}$ NMR $(300 \mathrm{MHz}$, $\left.\mathrm{CDCl}_{3}, 298 \mathrm{~K}\right) \delta_{\mathrm{H}} 7.36(1 \mathrm{H}, \mathrm{d}, J=7.8 \mathrm{~Hz}), 7.26(1 \mathrm{H}, \mathrm{d}, J=$ $7.8 \mathrm{~Hz}), 7.15(1 \mathrm{H}$, apparent $\mathrm{td}, J=7.7,1.2 \mathrm{~Hz}), 6.99(1 \mathrm{H}$, apparent td, $J=7.7,1.2 \mathrm{~Hz}), 3.69-3.62(4 \mathrm{H}, \mathrm{m}), 2.07-2.01$ 
$(4 \mathrm{H}, \mathrm{m}) ;{ }^{13} \mathrm{C}\left\{{ }^{1} \mathrm{H}\right\}$ NMR $\left(75 \mathrm{MHz}, \mathrm{CDCl}_{3}, 298 \mathrm{~K}\right) \delta_{\mathrm{c}} 160.9$, 148.9, 143.5, 123.6, 119.9, 115.8, 108.4, 47.2 (2C), 25.4 (2C); HRMS (ESI) $m / z$ : calculated for $[\mathrm{M}+\mathrm{H}]^{+}, \mathrm{C}_{11} \mathrm{H}_{13} \mathrm{~N}_{2} \mathrm{O}^{+}$, 189.1023, found: 189.1030 .

2-(Pyrrolidin-1-yl)naphtho[2,3-d] oxazole (2b) To a stirred solution of 2-(trichloromethyl)naphtho[2,3- $d]$ oxazole (1b, 158 $\mathrm{mg}, 0.55 \mathrm{mmol})$ in dry acetonitrile $(1 \mathrm{M})$ at r.t. was added pyrrolidine $(51 \mu \mathrm{L}, 0.6 \mathrm{mmol})$. The resultant solution was stirred at $60{ }^{\circ} \mathrm{C}$ for $4 \mathrm{~h}$. The solvent was removed in vacuo and the residue was purified by flash column chromatography on silica gel (5\% ethyl acetate in petroleum ether) to afford 2(pyrrolidin-1-yl)naphtho[2,3-d]oxazole (2b, $130 \mathrm{mg}, 99 \%$ yield) as a yellow oil. IR $\left(\mathrm{CHCl}_{3}\right) \mathrm{n}\left(\mathrm{cm}^{-1}\right) 2969,2881,1656$; ${ }^{1} \mathrm{H}$ NMR $\left(300 \mathrm{MHz}, \mathrm{CDCl}_{3}, 298 \mathrm{~K}\right) \delta_{\mathrm{H}} 7.86-7.80(2 \mathrm{H}, \mathrm{m})$, $7.68(1 \mathrm{H}, \mathrm{s}), 7.58(1 \mathrm{H}, \mathrm{s}), 7.37(2 \mathrm{H}$, quind, $J=6.9,1.3 \mathrm{~Hz})$, $3.66(4 \mathrm{H}, \mathrm{t}, J=6.7 \mathrm{~Hz}), 2.05-1.97(4 \mathrm{H}, \mathrm{m}) ;{ }^{13} \mathrm{C}\left\{{ }^{1} \mathrm{H}\right\} \mathrm{NMR}$ $\left(75 \mathrm{MHz}, \mathrm{CDCl}_{3}, 298 \mathrm{~K}\right) \delta_{\mathrm{c}} 161.9,149.3,144.1,131.9,129.2$, 127.6, 127.5, 124.2, 123.5, 111.4, 104.3, 47.5 (2C), 25.5 (2C); HRMS (ESI) $m / z$ : calculated for $[\mathrm{M}+\mathrm{H}]^{+}, \mathrm{C}_{15} \mathrm{H}_{15} \mathrm{~N}_{2} \mathrm{O}^{+}$, 239.1179, found: 239.1189 .

5-Methoxy-2-(pyrrolidin-1-yl)benzo[d] oxazole $(2 \mathrm{c})^{29}$ To a stirred solution of 5-methoxy-2-(trichloromethyl) benzo $[d]$ oxazole $(1 \mathrm{c}, 147 \mathrm{mg}, 0.55 \mathrm{mmol})$ in dry acetonitrile $(1 \mathrm{M})$ at r.t. was added pyrrolidine $(51 \mu \mathrm{L}, 0.6 \mathrm{mmol})$. The resultant solution was stirred at $60{ }^{\circ} \mathrm{C}$ for $4 \mathrm{~h}$. The solvent was removed in vacuo and the residue was purified by flash column chromatography on silica gel $(5 \%$ ethyl acetate in petroleum ether) to afford 5-methoxy-2-(pyrrolidin-1yl)benzo $[d]$ oxazole (2c, $83 \mathrm{mg}, 69 \%$ yield) as a light brown crystals. IR $\left(\mathrm{CHCl}_{3}\right) \mathrm{n}\left(\mathrm{cm}^{-1}\right) 2957,2880,1650 ;{ }^{1} \mathrm{H}$ NMR $(300$ $\left.\mathrm{MHz}, \mathrm{CDCl}_{3}, 298 \mathrm{~K}\right) \delta_{\mathrm{H}} 7.10(1 \mathrm{H}, \mathrm{d}, J=8.6 \mathrm{~Hz}), 6.92(1 \mathrm{H}, \mathrm{d}$, $J=2.5 \mathrm{~Hz}), 6.53(1 \mathrm{H}, \mathrm{dd}, J=8.6,2.5 \mathrm{~Hz}), 3.79(3 \mathrm{H}, \mathrm{s}), 3.64-$ $3.60(4 \mathrm{H}, \mathrm{m}), 2.04-1.99(4 \mathrm{H}, \mathrm{m}) ;{ }^{13} \mathrm{C}\left\{{ }^{1} \mathrm{H}\right\} \mathrm{NMR}(75 \mathrm{MHz}$, $\left.\mathrm{CDCl}_{3}, 298 \mathrm{~K}\right) \delta_{\mathrm{c}} 161.8,156.9,144.7,143.6,108.3,106.3$, $101.2,55.8,47.3$ (2C), 25.6 (2C); HRMS (ESI) $\mathrm{m} / z$ : calculated for $[\mathrm{M}+\mathrm{H}]^{+}, \quad \mathrm{C}_{12} \mathrm{H}_{15} \mathrm{~N}_{2} \mathrm{O}_{2}^{+}, 219.1129$, found: 219.1134 .

5-Chloro-2-(pyrrolidin-1-yl)benzo $[\boldsymbol{d}]$ oxazole $\quad(\mathbf{2 d})^{29}$ To a stirred solution of 5-chloro-2-(trichloromethyl) benzo[d]oxazole (1d, $150 \mathrm{mg}, 0.55 \mathrm{mmol})$ in dry acetonitrile $(1 \mathrm{M})$ at r.t. was added pyrrolidine $(51 \mu \mathrm{L}, 0.6 \mathrm{mmol})$. The resultant solution was stirred at $60{ }^{\circ} \mathrm{C}$ for $4 \mathrm{~h}$. The solvent was removed in vacuo and the residue was purified by flash column chromatography on silica gel $(5 \%$ ethyl acetate in petroleum ether) to afford 5-chloro-2-(pyrrolidin-1yl)benzo[d]oxazole (2d, $100 \mathrm{mg}, 81 \%$ yield) as a white solid. m.p. $121-123{ }^{\circ} \mathrm{C}$; IR $\left(\mathrm{CHCl}_{3}\right) \mathrm{n}\left(\mathrm{cm}^{-1}\right) 2979,2882,1650 ;{ }^{1} \mathrm{H}$ NMR $\left(300 \mathrm{MHz}, \mathrm{CDCl}_{3}, 298 \mathrm{~K}\right) \delta_{\mathrm{H}} 7.28(1 \mathrm{H}, \mathrm{d}, J=2.1 \mathrm{~Hz})$, $7.10(1 \mathrm{H}, \mathrm{d}, J=8.4 \mathrm{~Hz}), 6.90(1 \mathrm{H}, \mathrm{dd}, J=8.4,2.1 \mathrm{~Hz}), 3.60$ $(4 \mathrm{H}, \mathrm{t}, J=6.7 \mathrm{~Hz}), 2.05-1.96(4 \mathrm{H}, \mathrm{m}) ;{ }^{13} \mathrm{C}\left\{{ }^{1} \mathrm{H}\right\} \mathrm{NMR}(75$ $\left.\mathrm{MHz}, \mathrm{CDCl}_{3}, 298 \mathrm{~K}\right) \delta_{\mathrm{c}} 161.6,147.5,145.0,128.9,119.6$, $115.8,108.9,47.3$ (2C), 25.4 (2C); HRMS (ESI) $m / z$ : calculated for $[\mathrm{M}+\mathrm{H}]^{+}, \mathrm{C}_{11} \mathrm{H}_{12} \mathrm{ClN}_{2} \mathrm{O}^{+}, 223.0633$, found: 223.0637 .

6-Chloro-2-(pyrrolidin-1-yl)benzo $[\boldsymbol{d}]$ oxazole (2e) To a stirred solution of 6-chloro-2-(trichloromethyl) benzo[d]oxazole (1e, $150 \mathrm{mg}, 0.55 \mathrm{mmol})$ in dry acetonitrile $(1 \mathrm{M})$ at r.t. was added pyrrolidine $(51 \mu \mathrm{L}, 0.6 \mathrm{mmol})$. The resultant solution was stirred at $60{ }^{\circ} \mathrm{C}$ for $4 \mathrm{~h}$. The solvent was removed in vacuo and the residue was purified by flash column chromatography on silica gel $(5 \%$ ethyl acetate in petroleum ether) to afford 6-chloro-2-(pyrrolidin-1yl)benzo $[d]$ oxazole (2e, $93 \mathrm{mg}, 75 \%$ yield) as a white solid. m.p. $126-128{ }^{\circ} \mathrm{C}$; IR $\left(\mathrm{CHCl}_{3}\right) \mathrm{n}\left(\mathrm{cm}^{-1}\right) 2979,2881,1651 ;{ }^{1} \mathrm{H}$ NMR $\left(300 \mathrm{MHz}, \mathrm{CDCl}_{3}, 298 \mathrm{~K}\right) \delta_{\mathrm{H}} 7.22-7.19(2 \mathrm{H}, \mathrm{m}), 7.08$ - $7.00(1 \mathrm{H}, \mathrm{m}), 3.62-3.58(4 \mathrm{H}, \mathrm{m}), 2.05-1.96(4 \mathrm{H}, \mathrm{m})$; ${ }^{13} \mathrm{C}\left\{{ }^{1} \mathrm{H}\right\} \mathrm{NMR}\left(75 \mathrm{MHz}, \mathrm{CDCl}_{3}, 298 \mathrm{~K}\right) \delta_{\mathrm{c}} 161.2,149.1$, $142.6,125.0,124.0,116.1,109.3,47.4(2 \mathrm{C}), 25.5(2 \mathrm{C})$; HRMS (ESI) $m / z$ : calculated for $[\mathrm{M}+\mathrm{H}]^{+}, \mathrm{C}_{11} \mathrm{H}_{12} \mathrm{ClN}_{2} \mathrm{O}^{+}$, 223.0633, found: 223.0639 .

2-Methoxybenzo $[\boldsymbol{d}]$ oxazole (3a) ${ }^{30}$ To a stirred solution of 2(trichloromethyl)benzo[d] oxazole (1a, $150 \mathrm{mg}, 0.6 \mathrm{mmol}, 1$ equiv.) in acetonitrile $(0.6 \mathrm{~mL}, 1 \mathrm{M})$ at ambient temperature were added methanol $(28 \mu \mathrm{L}, 0.7 \mathrm{mmol}, 1.1$ equiv. $)$ and DBU (104 $\mu \mathrm{L}, 0.7 \mathrm{mmol}, 1.1$ equiv.). The resultant solution was stirred at $60{ }^{\circ} \mathrm{C}$ for $16 \mathrm{~h}$. The mixture was allowed to cool to r.t. and filtered through silica (dichloromethane). The solvent was removed in vacuo and the residue was purified by flash column chromatography on base washed silica gel (3\% ethyl acetate in petroleum ether) to afford 2methoxybenzo[ $[d]$ oxazole $(3 \mathrm{a}, 24 \mathrm{mg}, 25 \%)$ as a clear oil. $R_{\mathrm{f}}=$ 0.50 (1:2 ethyl acetate:petroleum); IR $\left(\mathrm{CHCl}_{3}\right) \mathrm{n}\left(\mathrm{cm}^{-1}\right) 3156$, 2927, 2254, 1792, 1747, 1552; ${ }^{1} \mathrm{H}$ NMR $\left(300 \mathrm{MHz}, \mathrm{CDCl}_{3}\right.$, $298 \mathrm{~K}) \delta_{\mathrm{H}} 7.90(1 \mathrm{H}, \mathrm{d} J=7.9 \mathrm{~Hz}) 7.67(1 \mathrm{H}, \mathrm{d} J=8.1 \mathrm{~Hz})$, $7.56-7.44(2 \mathrm{H}, \mathrm{m}), 4.10(3 \mathrm{H}, \mathrm{s}) ;{ }^{13} \mathrm{C}\left\{{ }^{1} \mathrm{H}\right\}$ NMR $(75 \mathrm{MHz}$, $\left.\mathrm{CDCl}_{3}, 298 \mathrm{~K}\right) \delta_{\mathrm{c}} 156.9,150.9,140.5,128.3,125.9,122.2$, $111.8,53.7$; HRMS (ESI) $\mathrm{m} / \mathrm{z}$ : calculated for $[\mathrm{M}+\mathrm{H}]^{+}$, $\mathrm{C}_{8} \mathrm{H}_{8} \mathrm{NO}_{2}^{+}, 150.0550$, found: 150.0540 .

2-(Benzyloxy)benzo $[\boldsymbol{d}]$ oxazole (3b) ${ }^{31}$ To a stirred solution of 2-(trichloromethyl)benzo[ $d]$ oxazole (1a, $150 \mathrm{mg}, 0.6 \mathrm{mmol}, 1$ equiv.) in acetonitrile $(0.6 \mathrm{~mL}, 1 \mathrm{M})$ were added benzyl alcohol $(73 \mu \mathrm{L}, 0.7 \mathrm{mmol}, 1.1$ equiv.) and DBU $(104 \mu \mathrm{L}, 0.7$ mmol, 1.1 equiv.). The resultant solution was stirred at $60{ }^{\circ} \mathrm{C}$ for $16 \mathrm{~h}$. The mixture was allowed to cool to r.t. and filtered through silica (dichloromethane). The solvent was removed in vacuo and the residue was purified by flash column chromatography on base washed silica gel (1\% ethyl acetate in petroleum ether) to afford 2-(benzyloxy)benzo[d]oxazole (3b, $37 \mathrm{mg}, 26 \%)$ as a clear oil. IR $\left(\mathrm{CHCl}_{3}\right) \mathrm{n}\left(\mathrm{cm}^{-1}\right) 3009,2928$, 2855, 1741, 1613, 1568; ${ }^{1} \mathrm{H}$ NMR $\left(300 \mathrm{MHz}, \mathrm{CDCl}_{3}, 298 \mathrm{~K}\right)$ $\delta_{\mathrm{H}} 7.81-7.79(2 \mathrm{H}, \mathrm{m}) 7.64-7.62(2 \mathrm{H}, \mathrm{m}), 7.47(2 \mathrm{H}$, apparent td, $J=7.7,1.4 \mathrm{~Hz}), 7.42-7.35(3 \mathrm{H}, \mathrm{m}), 6.87(2 \mathrm{H} \mathrm{s})$; ${ }^{13} \mathrm{C}\left\{{ }^{1} \mathrm{H}\right\} \mathrm{NMR}\left(75 \mathrm{MHz}, \mathrm{CDCl}_{3}, 298 \mathrm{~K}\right) \delta_{\mathrm{c}} 159.6,151.0$, $140.1,128.6,128.3,126.9$ (2C), 125.3 (2C), 121.2 (2C), 119.4, 111.3, 61.0; HRMS (ESI) $\mathrm{m} / \mathrm{z}$ : calculated for $[\mathrm{M}+\mathrm{H}]^{+}$, $\mathrm{C}_{14} \mathrm{H}_{12} \mathrm{NO}_{2}^{+}, 226.0863$, found: 226.0873 .

Benzo[d]oxazol-2-yl(pyrrolidin-1-yl)methanone (5) Method $A$ : To a stirred solution of 2-(trichloromethyl)benzo[ $d]$ oxazole (1a, $100 \mathrm{mg}, 0.42 \mathrm{mmol})$ in acetonitrile $(0.01 \mathrm{M})$ at ambient temperature was added pyrrolidine $(40 \mu \mathrm{L}, 0.47 \mathrm{mmol})$. The resultant solution was stirred at ambient temperature for $76 \mathrm{~h}$. The solvent was removed in vacuo and the residue was purified by flash column chromatography on silica gel $(5 \%$ ethyl acetate in diethyl ether) to afford benzo $[d]$ oxazol-2yl(pyrrolidin-1-yl)methanone $(5,60 \mathrm{mg}, 66 \%$ ) as a yellow solid. Method B: To a stirred solution of 2(trichloromethyl)benzo[d]oxazole (1a, $100 \mathrm{mg}, 0.42 \mathrm{mmol})$ in 
acetonitrile $(0.01 \mathrm{M})$ at ambient temperature was added pyrrolidine $(40 \mu \mathrm{L}, 0.47 \mathrm{mmol})$. The resultant solution was stirred at ambient temperature for $19 \mathrm{~h}$. The solvent was removed in vacuo and the residue was purified by flash column chromatography on silica gel (5\% ethyl acetate in diethyl ether) to afford benzo[ $[d]$ oxazol-2-yl(pyrrolidin-1yl)methanone $(\mathbf{5}, 32 \mathrm{mg}, 35 \%)$ as a yellow solid. Method $C$ : To a stirred solution of 2-(trichloromethyl)benzo[d]oxazole (1a, $100 \mathrm{mg}, 0.42 \mathrm{mmol})$ in acetonitrile $(0.01 \mathrm{M})$ at ambient temperature were added iron(III)chloride $(68 \mathrm{mg}, 0.42 \mathrm{mmol})$ and pyrrolidine $(40 \mu \mathrm{L}, 0.47 \mathrm{mmol})$. The resultant solution was stirred at ambient temperature for $19 \mathrm{~h}$. The solvent was removed in vacuo and the residue was purified by flash column chromatography on silica gel $(5 \%$ ethyl acetate in diethyl ether) to afford benzo[d]oxazol-2-yl(pyrrolidin-1yl)methanone (5, $52 \mathrm{mg}, 57 \%)$ as a yellow solid. m.p. 126-128 ${ }^{\circ} \mathrm{C} ; R_{\mathrm{f}}=0.20$ (1:9 ethyl acetate:petroleum ether) IR $\left(\mathrm{CHCl}_{3}\right)$ $\mathrm{n}\left(\mathrm{cm}^{-1}\right)$ 3006, 2886, 1640; ${ }^{1} \mathrm{H}$ NMR $\left(300 \mathrm{MHz}, \mathrm{CDCl}_{3}, 298 \mathrm{~K}\right)$ $\delta_{\mathrm{H}} 7.83(1 \mathrm{H}, \mathrm{d}, J=7.5 \mathrm{~Hz}) 7.66(1 \mathrm{H}, \mathrm{d}, J=7.6 \mathrm{~Hz}) 7.48-$ $7.42(2 \mathrm{H}, \mathrm{m}), 4.14(2 \mathrm{H}, \mathrm{t}, J=6.8 \mathrm{~Hz}), 3.76(2 \mathrm{H}, \mathrm{t}, J=6.8 \mathrm{~Hz})$, $2.11-1.93(4 \mathrm{H}, \mathrm{m}) ;{ }^{13} \mathrm{C}\left\{{ }^{1} \mathrm{H}\right\} \mathrm{NMR}\left(75 \mathrm{MHz}, \mathrm{CDCl}_{3}, 298 \mathrm{~K}\right)$ $\delta_{\mathrm{c}} 155.8,155.3,150.1,140.6,127.2,125.2,121.4,111.7,49.3$, 47.5, 26.5, 23.9; HRMS (ESI) $\mathrm{m} / z$ : calculated for $[\mathrm{M}+\mathrm{H}]^{+}$, $\mathrm{C}_{12} \mathrm{H}_{13} \mathrm{~N}_{2} \mathrm{O}_{2}^{+}, 217.0972$, found: 217.0970 .

\section{6-Chloro-2,3-di(pyrrolidin-1-yl)-2H-benzo $[b][1,4]$ oxazine}

(6d) To a stirred solution of 5-chloro-2-(trichloromethyl) benzo $[d]$ oxazole $(\mathbf{1 d}, 150 \mathrm{mg}, 0.55 \mathrm{mmol})$ in dry acetonitrile $(1 \mathrm{M})$ at r.t. was added pyrrolidine $(51 \mu \mathrm{L}, 0.6 \mathrm{mmol})$. The resultant solution was stirred at $60^{\circ} \mathrm{C}$ for $4 \mathrm{~h}$. The solvent was removed in vacuo and the residue was purified by flash column chromatography on silica gel $(5 \%$ ethyl acetate in petroleum ether) to afford 6-chloro-2,3-di(pyrrolidin-1-yl)- $2 \mathrm{H}$ benzo[b][1,4]oxazine (6d, $24 \mathrm{mg}, 14 \%$ yield) as a yellow gummy solid. IR $\left(\mathrm{CHCl}_{3}\right) \mathrm{n}\left(\mathrm{cm}^{-1}\right) 3690,2929,2876,1610 ;{ }^{1} \mathrm{H}$ NMR $\left(300 \mathrm{MHz}, \mathrm{CDCl}_{3}, 298 \mathrm{~K}\right) \delta_{\mathrm{H}} 7.08(1 \mathrm{H}, \mathrm{d}, J=2.1 \mathrm{~Hz})$, $6.80-6.72(2 \mathrm{H}, \mathrm{m}), 5.45(1 \mathrm{H}, \mathrm{s}), 3.65(3 \mathrm{H}, \mathrm{br} . \mathrm{s}), 3.36(1 \mathrm{H}$, br.s) $2.96-2.89(2 \mathrm{H}, \mathrm{m}) 2.68-2.61(2 \mathrm{H}, \mathrm{m}) 1.97$ (4H, br.s) $1.72-1.60(4 \mathrm{H}, \mathrm{m}) ;{ }^{13} \mathrm{C}\left\{{ }^{1} \mathrm{H}\right\} \mathrm{NMR}\left(75 \mathrm{MHz}, \mathrm{CDCl}_{3}, 298 \mathrm{~K}\right)$ $\delta_{\mathrm{c}} 152.3,144.7,136.0,125.8,123.3,121.9,115.0,80.2,46.7$ (4C), 24.4 (4C); HRMS (ESI) $\mathrm{m} / \mathrm{z}$ : calculated for $[\mathrm{M}+\mathrm{H}]^{+}$, $\mathrm{C}_{16} \mathrm{H}_{21} \mathrm{ClN}_{3} \mathrm{O}^{+}, 306.1368$, found: 306.1373 .

\section{7-Chloro-2,3-di(pyrrolidin-1-yl)-2H-benzo[b][1,4]oxazine}

(6e) To a stirred solution of 6-chloro-2-(trichloromethyl) benzo $[d]$ oxazole $(1 \mathrm{e}, 150 \mathrm{mg}, 0.55 \mathrm{mmol})$ in dry acetonitrile (1 M) at r.t. was added pyrrolidine $(51 \mu \mathrm{L}, 0.6 \mathrm{mmol})$. The resultant solution was stirred at $60{ }^{\circ} \mathrm{C}$ for $4 \mathrm{~h}$. The solvent was removed in vacuo and the residue was purified by flash column chromatography on silica gel $(5 \%$ ethyl acetate in petroleum ether) to afford 7-chloro-2,3-di(pyrrolidin-1-yl)- $2 \mathrm{H}$ benzo $[b][1,4]$ oxazine $(6 \mathbf{e}, 42 \mathrm{mg}, 25 \%$ yield) as a yellow gummy solid. IR $\left(\mathrm{CHCl}_{3}\right) \mathrm{n}\left(\mathrm{cm}^{-1}\right) 3667,2974,2876,1607 ;{ }^{1} \mathrm{H}$ NMR $\left(300 \mathrm{MHz}, \mathrm{CDCl}_{3}, 298 \mathrm{~K}\right) \delta_{\mathrm{H}} 7.01(1 \mathrm{H}, \mathrm{dd}, J=7.9,0.7$ $\mathrm{Hz}), 6.84-6.80(2 \mathrm{H}, \mathrm{m}), 5.46(1 \mathrm{H}, \mathrm{s}), 3.63$ (3H, br.s), 3.37 (1H, br.s) $2.96-2.88(2 \mathrm{H}, \mathrm{m}) 2.68-261(2 \mathrm{H}, \mathrm{m}) 2.01-1.94$ $(4 \mathrm{H}, \mathrm{m}) 1.69-1.60(4 \mathrm{H}, \mathrm{m}) ;{ }^{13} \mathrm{C}\left\{{ }^{1} \mathrm{H}\right\} \mathrm{NMR}\left(75 \mathrm{MHz}, \mathrm{CDCl}_{3}\right.$, $298 \mathrm{~K}) \delta_{\mathrm{c}} 151.8,146.5,133.5,126.6,124.1,121.3,114.6$, 80.2, 46.6 (4C), 24.4 (4C); HRMS (ESI) $\mathrm{m} / \mathrm{z}$ : calculated for $[\mathrm{M}+\mathrm{H}]^{+}, \mathrm{C}_{16} \mathrm{H}_{21} \mathrm{ClN}_{3} \mathrm{O}^{+}, 306.1368$, found: 306.1360 .
2,2,2-Trichloro- $N$-(2-hydroxyphenyl) acetamide $\quad(7)^{32}$ Method A: To a stirred solution of 2(trichloromethyl)benzo[d]oxazole (1a, 237mg, $1 \mathrm{mmol}, 1$ equiv.) and iron(III) chloride (3.0 $\mathrm{mg}, 0.02 \mathrm{mmol}, 2 \mathrm{~mol} \%$ ) in acetonitrile $(0.2 \mathrm{~mL}, 5 \mathrm{M})$ at r.t. was added water $(18 \mu \mathrm{L}, 1$ mmol, 1.0 equiv.). The resultant mixture was stirred at r.t. for $5 \mathrm{~h}$. Water $(27 \mu \mathrm{L}, 1.5 \mathrm{mmol}, 1.5$ equiv.) was added. The mixture was stirred at r.t, for $3 \mathrm{~h}$. The solvent was removed in vacuo and the residue was purified by flash column chromatography on silica gel $(5 \%$ ethyl acetate in petroleum ether) to afford 2,2,2-trichloro- $N$-(2-hydroxyphenyl) acetamide $(7,148 \mathrm{mg}, 58 \%)$ as a yellow solid. Method B: 2(Trichloromethyl)-benzo[ $d]$ oxazole (1a, $61 \mathrm{mg}, 0.26 \mathrm{mmol})$ in $\mathrm{H}_{2} \mathrm{O}(1.0 \mathrm{~mL})$ was stirred at $150{ }^{\circ} \mathrm{C}$ for $2 \mathrm{~h}$. The solution was allowed to cool to r.t. and dichloromethane $(10 \mathrm{ml})$ was added. The resultant solution was washed with water $(3 \times 5 \mathrm{~mL})$. The organic layer was dried over magnesium sulfate and the solvent was removed in vacuo to afford 2,2,2-trichloro- $\mathrm{N}$-(2hydroxyphenyl) acetamide $(7,24 \mathrm{mg}, 37 \%)$ as a yellow solid. m.p. $160-161{ }^{\circ} \mathrm{C}$ (lit. m.p. $\left.160{ }^{\circ} \mathrm{C}\right)$; IR $\left(\mathrm{CHCl}_{3}\right) \mathrm{n}\left(\mathrm{cm}^{-1}\right) 3594$, 3397, 1720, 1615; ${ }^{1} \mathrm{H}$ NMR $\left(300 \mathrm{MHz}, \mathrm{CDCl}_{3}, 298 \mathrm{~K}\right) \delta_{\mathrm{H}} 8.98$ (1H, br.s) $7.95(1 \mathrm{H}, \mathrm{d}, J=8.0 \mathrm{~Hz}), 7.13-7.09(1 \mathrm{H}, \mathrm{m}), 7.02$ - 6.93 (2H, m) 5.99 (1H br.s); ${ }^{13} \mathrm{C}\left\{{ }^{1} \mathrm{H}\right\}$ NMR $(75 \mathrm{MHz}$, $\left.\mathrm{CDCl}_{3}, 298 \mathrm{~K}\right) \delta_{\mathrm{c}} 159.7,146.1,126.6,124.5,121.7,121.2$, 116.5 , 92.5; HRMS (ESI) $\mathrm{m} / \mathrm{z}$ : calculated for $[\mathrm{M}+\mathrm{H}]^{+}$, $\mathrm{C}_{8} \mathrm{H}_{7} \mathrm{Cl}_{3} \mathrm{NO}_{2}{ }^{+}, 253.9537$, found: 253.9534 .

(Z)-2,2'-(1-Chloro-2-(pyrrolidin-1-yl)ethene-1,2diyl)bis(benzo $[\boldsymbol{d}]$-oxazole) (8) To a stirred suspension of 2(trichloromethyl)benzo[d] oxazole (1a, $150 \mathrm{mg}, 0.6 \mathrm{mmol}, 1.0$ equiv.) and iron(III) chloride (97mg, $0.6 \mathrm{mmol}, 1.0$ equiv.) in acetonitrile $(6 \mathrm{~mL}, 0.1 \mathrm{M})$ at r.t was added pyrrolidine $(260$ $\mu \mathrm{L}, 3.17 \mathrm{mmol}, 5.0$ equiv.). The resultant mixture was stirred at r.t for $19 \mathrm{~h}$ and then filtered through base washed silica. The solvent was removed in vacuo and the residue was purified by flash column chromatography on silica gel $(5 \%$ diethyl ether in petroleum ether) to afford (Z)-2,2'-(1-chloro-2-(pyrrolidin1-yl)ethene-1,2-diyl)bis(benzo[ $d]$ oxazole) $(8,58 \mathrm{mg}, 26 \%)$ as a yellow solid. $R_{\mathrm{f}}=0.40$ (1:9 ethyl acetate:petroleum);IR $\left(\mathrm{CHCl}_{3}\right) \mathrm{n}\left(\mathrm{cm}^{-1}\right) 2982,2882,1607,1578,1549 ;{ }^{\mathrm{H}} \mathrm{NMR}(300$ $\left.\mathrm{MHz} \mathrm{CDCl}_{3}, 298 \mathrm{~K}\right) \delta_{\mathrm{H}} 7.80-7.77(1 \mathrm{H}, \mathrm{m}), 7.56-7.54(1 \mathrm{H}$, $\mathrm{m}), 7.44-7.39(3 \mathrm{H}, \mathrm{m}), 7.13(1 \mathrm{H}$, apparent td, $J=7.7,1.1 \mathrm{~Hz}$ ), $7.05(1 \mathrm{H}$, apparent td, $J=7.8,1.3 \mathrm{~Hz}), 6.87(1 \mathrm{H}, \mathrm{d}, J=7.8$ $\mathrm{Hz}), 3.66-3.62(4 \mathrm{H}, \mathrm{m}), 2.00-1.97(4 \mathrm{H}, \mathrm{m}) ;{ }^{13} \mathrm{C}\left\{{ }^{1} \mathrm{H}\right\} \mathrm{NMR}$ $\left(75 \mathrm{MHz}, \mathrm{CDCl}_{3}, 298 \mathrm{~K}\right) \delta_{\mathrm{c}} 161.8,158.1,150.5,150.4,141.9$, $141.2,138.6,126.1,124.8,124.1,124.0,120.9,119.3,111.1$, $109.5,94.7,51.3$ (2C), 25.6 (2C); HRMS (ESI) $m / z$ : calculated for $[\mathrm{M}+\mathrm{H}]^{+}, \mathrm{C}_{20} \mathrm{H}_{17} \mathrm{ClN}_{3} \mathrm{O}_{2}{ }^{+}, 366.1004$, found: 366.1000 .

ACKNOWLEDGMENT The School of Chemistry at the University of Nottingham, the Department of Chemical Sciences at the University of Huddersfield and a Royal Society Research Grant (RG130535) supported this work.

SUPPORTING INFORMATION Tables of reaction parameters examined and copies of ${ }^{1} \mathrm{H}$ and ${ }^{13} \mathrm{C}$ NMR spectra of all newly synthesized products as well as X-ray details of compounds $\mathbf{6 e}, 7$ and 8. This material is available free of charge via the Internet at http://pubs.acs.org. 
${ }^{1}$ (a) Liu, K. G.; Lo, J. R.; Comery, T. A.; Zhang, G. M.; Zhang, J. Y.; Kowal, D. M.; Smith, D. L.; Di, L.; Kerns, E. H.; Schechter, L. E.; Robichaud, A. Bioorg. Med. Chem. Lett. 2009, 19, 1115-1117. (b) Sheng, C.; Xu, H.; Wang, W.; Cao, Y.; Dong, G.; Wang, S.; Che, X.; Ji, H.; Miao, Z.; Yao, J.; Zhang, W. Eur. J. Med. Chem. 2010, 45, 3531-3539. (c) Cox, C. D.; Breslin, M. J.; Whitman, D. B.; Schreier, J. D.; McGaughey, G. B.; Bogusky, M. J.; Roecker A. J.; Mercer, S. P.; Bednar, R. A.; Lemaire, W.; Bruno, J. G.; Reiss, D. R.; Harrell, C. M.; Murphy, K. L.; Garson, S. L.; Doran, S. M.; Prueksaritanont, T.; Anderson, W. B.; Tang, C.; Roller, S.; Cabalu, T. D.; Cui, D.; Hartman, G. D.; Young, S. D.; Koblan, K. S.; Winrow, C. J.; Renger, J. J.; Coleman, P.J. J Med. Chem. 2010, 53, 5320-5332. (d) Lo, W. C.; Hunter, J. E.; Watson, G. B.; Patny, A.; Iyer, P. S.; Boruwa, J. U.S. Patent 20160058010, November 3, 2015. ${ }^{2}$ For recent examples, see: (a) Más-Montoya, M.; Usea, L.; Ferao, A. E.; Montenegro, M. F.; Ramírez de Arellano, C.; Tárraga, A.; Rodríguez-López, J. N.; Curiel, D. J. Org. Chem. 2016, 81, 3296-3302. (b) Jiang, J.; Zou, H.; Dong, Q.; Wang, R.; Lu, L. Zhu, Y.; He, W. J. Org. Chem. 2016, 81, 51-56. (c) Aljaar, N.; Malakar, C. C.; Conrad, J.; Beifuss, U. J. Org. Chem. 2015, 80, 10829-10837. (d) Nimnual, P.; Tummatorn, J.; Thongsornkleeb, C.; Ruchirawat, S. J. Org. Chem. 2015, 80, 8657-8667. (e) Song, X.-R.; Qiu, Y. F.; Hao, X.-H.; Han, Y.-P.; Gao, P.; Liu, X.-Y.; Liang, Y.-M. J. Org. Chem 2015, 80, 2263-2271. (f) Khalafi-Nezhad, A.; Panahi, F. ACS Catal. 2014, 4 1686-1692. (g) Hosseini-Sarvari, M.; Khanivar, A.; Moeini, F. J. Mater. Sci. 2015, 50, 3065-3074. (h) Gao, W.-J.; Li, W.-C.; Zeng, C.-C.; Tian, H.-Y.; Hu, L.-M.; Little, R. D. J. Org. Chem. 2014, 79, 9613-9618. (i) Garduño, J. A.; García, J. J. ACS Catal. 2015, 5, 3470-3477. (j) Gu, L.; Jin, C.; Gua, J.; Zhang, L. Wang, W. Chem. Comm. 2013, 49, 10968-10970. (k) Gu, L/; Wang, W.; Xiong, Y.; Huang, X.; Li, G. Eur. J. Org. Chem. 2014, 319-322.

${ }^{3}$ For recent examples, see: (a) Lipp, A.; Lahm, G.; Opatz, T. J. Org. Chem 2016, 81, 4890-4897. (b) Smyth, L. A.; Phillips, E. M.; Chan, V. S. Napolitano, J. G.; Henry, R.; Shekhar, S. J. Org. Chem. 2016, 81, 1285-1294. (c) Yang, K.; Chen, X.; Wang, Y.; Li, W.; Kadi, A. A.; Fun, H.-K.; Sun, H.; Zhang, Y.; Li, G.; Lu, H. J. Org. Chem. 2015, 80, 11065-11072. (d) Yoon, H.; Lee, Y. J. Org. Chem. 2015, 80, 10244-10251. (e) Kim, D.; Yoo, K.; Kim, S. E.; Cho, H. J.; Lee, J.; Kim, Y.; Kim, M. J. Org. Chem. 2015, 80, 3670-3676. ${ }^{4}$ For the synthesis of 2-aminobenzoxazoles from activated starting materials, see: (a) Kumar, R. U.; Reddy, H. V.; Kumar, B. S. P. A.; Satish, G.; Reddy, V. P.; Nageswar, Y. V. D. Tetrahedron Lett. 2016, 57, 637-640. (b) Manolikakes, G.; Cavryushin, A.; Knochel, P. J. Org. Chem. 2008, 73, 1429-1434. (c) Cioffi, C. L.; Lansing, J. L.; Yüksel, H. J. Org. Chem. 2010, 75, 7942-7945.

5 For the synthesis of 2-amidobenzoxazoles from activated starting materials, see: (a) Zhang, Z.; Yin, Z.; Kadow, J. F.; Meanwell, N. A. Wang, T. J. Org. Chem. 2004, 69, 1360-1363. (b) Boger, D. L.; Schmitt, H. W.; Fink, B. E.; Hedrick, M. P. J. Org. Chem. 2001, 66, 6654-6661. (c) Stover, J. S.; Shi, J.; Jin, W.; Vogt, P. K.; Boger, D. L. J. Am. Chem. Soc. 2009, 131, 3342-3348. (d) Shin, S.; Kim, Y.; Kim, K.; Hong, S. Org. Biomol. Chem. 2014, 12, 5719 5726 .

${ }^{6}$ For the synthesis of 2-aminobenzoxazoles from unactivated starting materials via $\mathrm{C}-\mathrm{H}$ activation, see: (A) Froehr, T.; Sindlinger, C. P.; Kloechner, U. Finkbeiner, P.; Nachtsheim, B. J. Org. Lett. 2011, 13, 3754-3757. (b) Joseph, J.; Kim, J. Y.; Chang, S. Chem. Eur. J. 2011, 17, 8294-8298. (c) Wang, J.; Hou, J.-T.; Wen, J.; Zhang, J.; Yu, X.-Q. Chem. Comm. 2011, 47, 3652-3654 (d) Chen, S.; Zheng, K.; Chen, F. Tetrahedron Lett. 2012, 53, 6297-6299. (e) Xie, Y.; Qian, Bo, Xie, P.; Huang, H. Adv. Synth. Catal. 2013, 355, 13151322. (f) Wertz, S.; Kodama, S.; Studer, A.; Angew. Chem. Int. Ed. 2011, 50, 11511-11515. (g) Xu, D.; Wang, W.; Miao, C.; Zhang, Q.; Xia, C.; Sun, W. Green Chem. 2013, 15, 2975-2980. (h) Armstrong, A.; Collins, J. C. Angew. Chem. Int. Ed. 2010, 49, 2282-2285.

${ }^{7}$ For the synthesis of 2-amidobenzoxazoles from unactivated starting materials via C-H activation, see: He, Y.; Li, H.; Li, P.; Wang, L. Chem. Comm. 2011 47, 8946-8948

${ }^{8}$ (a) Zuo, W.; Lough, A. J.; Li, Y. F.; Morris, R. H. Science 2013, 342, 1080 1083 (b) Friedfeld, M. R.; Shevlin, M.; Hoyt, J. M.; Krska, S. W.; Tudge, M. T.; Chirik, P. J. Science 2013, 342, 1076-1080 (c) Bullock, R. M. Science 2013, 342, 1054-1055.

For recent reviews, see: (a) Allais, C.; Grassot, J.-M.; Rodriguez, J.; Constantieux, T. Chem. Rev. 2014, 114, 10829-10868. (b) Sun, C.-L; Shi, Z.-J. Chem. Rev. 2014, 114, 9219-9280.

For recent examples of the reactivity of trichloroacetonitrile, see: (a) Dunsford, J. J.; Camp, J. E. Tetrahedron Lett. 2013, 54, 4522-4523. (b) Lester, R. P.; Dunsford, J. J.; Camp, J. E. Org. Biomol. Chem. 2013, 11, 7472-7476. ${ }^{11}$ Lester, R. P.; Camp, J. E. ACS Sustainable Chem. Eng. 2013, 1, 545-548. 12 For a review, see: Camp, J. E. Eur. J. Org. Chem. 2016 DOI:10.1002/ejoc.201600803.

${ }^{13}$ For recent examples, see: (a) Mougenot, P.; Namane, C.; Fett, E.; Goumy, F.; Dadji-Faïhun, R.;Langot, G.; Monseau, C.; Onofri, B.; Pacquet, F.; Pascal, C.; Crespin, O.; Ben-Hassine, M.; Ragot, J.-L.: Van-Pham, T.; Philippo, C.; Chatelain-Egger, F.; Péron, P.; Le Bail, J.-C.; Guillot, E.; Chamiot-Clerc, P.;
Chabanaud, M.-A.; Pruniaux, M.-P.; Ménegotto, J.; Schmidt, F.; Venier, O, Viviani, F.; Nicolai, E. Bioorg. Med. Chem. Lett. 2016, 26, 25-32. (b) Bai, S. A.; Tangeda, S. J.; Madhavi, M.; Garlapati, A. Ind. J. Heterocyclic Chem. 2014, 24, 87-92. (c) Nanda, K. K.; Henze, D. A.; Penna, K. D.; Desai, R.; Leitl, M.; Lemaire, W.; White, R. B.; Yeh, S.; Brouillette, J. N.; Hartman, G. D.; Bilodeau, M T.; Trotter, B. W.; Bioorg. Med. Chem. Lett. 2014, 24, 1218 1221. (d) Staben, S. T.; Feng, J. A.; Lyle, K.; Belvin, M.; Boggs, J.; Burch, J. D.; Chua, C. C.; Cui, H.; DiPasquale, A. G.; Friedman, L. S.; Heise, C.; Koeppen, $\mathrm{H}$; Kotey, A; Mintzer, R.; Oh, A; Roberts, D. A.; Rouge, L Rudolph, J.; Tam, C.; Wang, W.; Xiao, Y.; Young, A.; Zhang, Y.; Hoeflich, K. P.J. Med. Chem. 2014, 57, 1033-1045. (e) Hemming, K. J. Chem. Res. 2001 209-216.

${ }^{4}$ Carruthers, N. I.; Dvorak, C. A.; Edwards, J. P.; Grice, C. A.; Jablonowski, J. A.; Ly, K. S.; Pio, B. A.; Shah, C. R.; Venable, J. D. WO2004022060 A2, September 6, 2002.

${ }^{5}$ For related additions of amines to 2-trichloromethylazoles, see: (a) Hoeflich, K. P.; Lyle, K. S.; Staben, S.; Schirlin, J. WO/2014/170421, October 23, 2014. (b) McLure, K. G.; Yiung, P. R. WO2013186612 A1, October 24, 2013. (c) Zhang, X.; Hufnagel, H. R.; Cai, C.; Lanter, J.; Markotan, T. Sui, Z. WO/2010/121011. (d) Jonathan, R.; Hatley, D.; Heer, J. P.; Liddle, J.; Mason, A. M.; Pinto, I. L.; Rahman, S. S.; Smith, I. E. D. WO2007017262 A1, February 15, 2007. (e) Eloy, F.; Lenaers, R. Helv. Chim. Acta. 1966, 49, 1430 1432.

${ }^{16}$ See ESI Tables S1-S5 for details.

For recent examples of nucleophilic catalysis, see: (a) Chung, W. Lindovská, P.; Camp, J. E. Tetrahedron Lett. 2011, 52, 6785-6787. (b) Ngwerume, S.; Camp, J. E. J. Org. Chem. 2010, 75, 6271-6274. (c) Ngwerume, S.; Lewis, W.; Camp, J. E. J. Org. Chem. 2013, 78, 920-934. ${ }_{18}^{18}$ See ESI Scheme S1.

For a similar degradation process of chloroform, see: Moenig, J.; Bahnemann, D.; Asmus, K. D. Chemico-Bio. Int. 1983, 47, 15

${ }^{20}$ For related additions of alcohols to 2-trichloromethylazoles, see: (a) Staben S.; Feng, J.; Loke, P. L.; Montalbetti, C. A. G. N. US20120214762 A1, August 23, 2012. (b) Bolton, R. E.; Coote, S. J.; Finch, H.; Lowdon, A.; Pegg, N.; Vinader, M. V. Tetrahedron Lett. 1995, 36, 4471-4474. (c) Yurugi, S.; Miyake, A.; Fushim, T.; Imamiya, E.; Matsumura, H.; Imai, Y. Chem. Pharm. Bull. 1973, 21, 1641-1650.

${ }^{21}$ For related amide forming processes with 2-trichloromethylazoles, see: (a) Reddy, K. B.; Murahari, K. K.; Kanakam, S.; Rajan, K. S.; Rao, G. M.; Kumar, B. V. World J. Pharm. Pharm. Sci. 2014, 3, 2017-2025. (b) Ahn, K.; Boehm, M.; Cabral, S.; Carpino, P. A.; Futatsugi. K.; Hepworth, D.; Kung, D. W.; Orr, S.; Wang, J. WO2013150416 A1, October 10, 2013. (c) Ellis, D. Synthetic Comm. 2009, 39, 2585-2595. (d) Venable, J. D.; Cai, H.; Chai, W.; Dvorak, C. A.; Grice, C. A.; Jablonowski, J. A.; Chah, C. R.; Kwok, A. K.; Ly, K. S.; Pio, B.; Wei, J.; Desai, P. J.; Jiang, W.; Nguyen, S.; Ling, P.; Wilson, S. J.; Dunford, P. J.; Thurmond, R. L.; Lovenburg, T. W.; Karlsson, L.; Carruthers, N. I.; Edwards, J. P. J. Med. Chem. 2005, 48, 8289-8298. (e) Song, J.; Ma, J.; Rabbat, C. J.; Nashashibi, I.; Chen, X.; Zhao, Z. US20120184572 A1, July 19, 2012.

${ }^{22}$ See ESI for details.

${ }^{23}$ Gauß, W.; Heitzer, H. Justus Liebigs Ann. Chem. 1970, 733, 59-69.

${ }^{24}$ Shafer, C. M.; Morse, D. I.; Molinski, T. F. Tetrahedron 1996, 52, 14475 14486.

${ }^{25}$ Van den Akker, L. W.; Brink, M. US5981803 A, November 9, 1999.

${ }^{26}$ Bravo, H. R.; Villarroel, E.; Copaja, S. V.; Argandoña, V. H. Z Naturforsch C 2008, 63, 389-394.

${ }^{27}$ For reviews on diversity-oriented synthesis (DOS), see: (a) Spring, D. Org. Biomol. Chem. 2003, 1, 3867-3870. (b) K. M. G. O'Connell, W. R. J. D Galloway and D. R. Spring, in Diversity-Oriented Synthesis, John Wiley \& Sons, Inc., 2013, 1-26.

${ }^{28}$ Manolikakes, G.; Gavryushin, A.; Knochel, P. J. Org. Chem. 2008, 73, 14291434.

${ }^{29}$ Joseph, J.; Kim, J. Y.; Chang, S. Chem.-Eur. J. 2011, 17, 8294-8298.

${ }^{30}$ Cioffi, C. L.; Lansing, J. J.; Yüksel, H. J. Org. Chem. 2010, 75, 7942-7945.

${ }^{31}$ Yamato, M.; Takeuchi, Y.; Hattori, K.; Hashigaki, K. Chem. Pharma. Bull. 1983, 31, 3946-3950.

${ }^{32}$ Spiegel, L.; Spiegel, P. Chem. Ber. 1907, 40, 1734. 